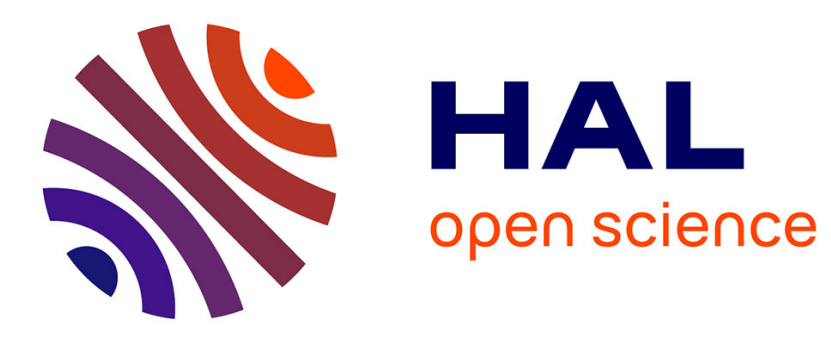

\title{
Lipomatose cervico-dorsale révélant un syndrome MERRF (myoclonic epilepsy with ragged red fibers)
}

\author{
F. Carré, R. Hervochon, C. Foirest, F. Tankéré
}

\section{To cite this version:}

F. Carré, R. Hervochon, C. Foirest, F. Tankéré. Lipomatose cervico-dorsale révélant un syndrome MERRF (myoclonic epilepsy with ragged red fibers). Annales françaises d'Oto-rhino-laryngologie et de Pathologie Cervico-faciale, 2019, 136, pp.115 - 117. 10.1016/j.aforl.2018.04.009 . hal-03486513

\section{HAL Id: hal-03486513 \\ https://hal.science/hal-03486513}

Submitted on 20 Dec 2021

HAL is a multi-disciplinary open access archive for the deposit and dissemination of scientific research documents, whether they are published or not. The documents may come from teaching and research institutions in France or abroad, or from public or private research centers.
L'archive ouverte pluridisciplinaire HAL, est destinée au dépôt et à la diffusion de documents scientifiques de niveau recherche, publiés ou non, émanant des établissements d'enseignement et de recherche français ou étrangers, des laboratoires publics ou privés.

\section{다)(1) $(5$}

Distributed under a Creative Commons Attribution - NonCommerciall 4.0 International 
Lipomatose cervico-dorsale révélant un syndrome MERRF (Myoclonic Epilepsy with Ragged Red Fibers)

Fabienne CARRE*, Rémi HERVOCHON, Claire FOIREST, Frédéric TANKERE

Service d'ORL et chirurgie cervico-faciale, AP-HP, Hôpital Pitié-Salpêtrière, service ORL, 75013 Paris, France - Sorbonne Université, Faculté de Médecine

* Auteur correspondant :

Carrefabienne@hotmail.com (Fabienne CARRE)

0623593925

$+33623593925$ 


\section{Résumé}

Introduction: Les patients atteints d'un syndrome MERRF (Myoclonic Epilepsy with Ragged Red Fibers) présentent principalement une encéphalo-myopathie. La lipomatose, rarement présente, est de localisation cervico-dorsale, progressive et récidivante.

$\underline{\text { Cas clinique }}$ : Nous rapportons 4 cas cliniques présentant l'association d'une lipomatose au syndrome MERRF. Chez 3 patients, le diagnostic de syndrome MERRF a pu être réalisé du fait de la présentation clinique lipomateuse et grâce à l'interrogatoire retrouvant des antécédents personnels ou familiaux de lipomes et de myopathie.

Discussion: La présence d'une lipomatose axiale extensive doit faire rechercher d'autres signes cliniques du syndrome MERRF chez le patient ou sa famille. Les enjeux diagnostiques sont liés au conseil génétique.

\section{Mots-clés :}

Syndrome MERRF

Lipomatose

Myopathie mitochondriale 


\section{Introduction}

Le syndrome MERRF (Myoclonic Epilepsy with Ragged Red Fibers) ou épilepsie myoclonique à fibres rouges déchiquetées, est une encéphalo-myopathie mitochondriale rare. Quelques cas se présentent avec une lipomatose cervico-thoracique qui peut être révélatrice de la maladie.

Nous présentons les cas rares d'une famille présentant des lipomes géants, et d'une patiente présentant une lipomatose, associés au syndrome MERRF.

L'objectif de cet article est de souligner l'association d'une lipomatose cervico-dorsale au syndrome MERRF, et les enjeux de dépistage et de prise en charge qui en résultent.

\section{Observations}

Les 3 premières observations concernent une fratrie portant la mutation la plus fréquente du syndrome MERRF : 8344A>G dans le gène MTTK de transfert de la lysine. Seul un patient était diagnostiqué lors de la première consultation. L'interrogatoire a retrouvé la présence de lipomes multiples chez la mère, ainsi que chez quelques oncles et tantes maternels, sans autres signes cliniques du syndrome MERRF.

$\mathrm{La} 4{ }^{\text {ème }}$ observation concerne une patiente présentant une mutation pathogénique variante (8363 G>A), non diagnostiquée lors de la consultation initiale. L'interrogatoire a révélé la présence de 2 cas de lipomatose dans la fratrie dont un associé à une myopathie.

\section{Observation $n^{\circ} 1$}

Le patient âgé de 50 ans présentait un lipome géant dorsal, associé à une lipomatose cervico-thoracique évoluant depuis 20 ans. Il avait comme antécédent une mitochondriopathie MERRF, associant myopathie et syndrome cérébelleux. L'exérèse chirurgicale a débuté par l'ablation du plus volumineux lipome dorsal (Fig. 1). La pièce opératoire pesait 3,5 kg. Les suites chirurgicales ont été compliquées d'un épanchement séreux, nécessitant une reprise chirurgicale au $15^{\text {ème }}$ jour postopératoire. L'exérèse des autres lipomes fut réalisée sans complication.

A distance, l'évolution clinique a été marquée par une récidive lipomateuse sur les sites d'exérèse chirurgicale.

\section{Observation $\mathbf{n}^{\circ} 2$}

Le patient âgé de 60 ans a consulté pour l'ablation de multiples lipomes cervicaux dont le plus volumineux mesurait $15 \mathrm{~cm}$ de grand axe. Il ne présentait pas d'autres signes cliniques du syndrome MERRF.

\section{Observation $\mathbf{n}^{\circ} 3$}

La patiente âgée de 40 ans présentait initialement un lipome dorsal de $12 \mathrm{~cm}$ de grand axe. Elle a développé secondairement d'autres lipomes cervico-dorsaux, certains étant des récidives de localisations antérieurement traitées chirurgicalement. La patiente présentait également une surdité, des troubles de la marche et des douleurs musculaires.

\section{Observation $n^{\circ} 4$}

La patiente âgée de 54 ans avait comme antécédents des troubles de la marche depuis l'enfance, une neuropathie sensitive et une hypoacousie bilatérale. Elle présentait 3 lipomes, de localisation sous-mentale, basi-cervicale et nucale, apparus vers l'âge de 40 ans.

L'évolution a été marquée par une récidive et de nouvelles localisations lipomateuses, de petite taille, dans les régions sous-mentale, sus-claviculaires, thoracique postérieure et nucale. 


\section{Discussion}

Les lipomes forment des masses sous-cutanées molles, compressibles, mobiles par rapport au plan profond, ne dépassant pas $5 \mathrm{~cm}$ dans $80 \%$ des cas. Ils apparaissent principalement chez les adultes de 40 à 60 ans, et sont multiples dans 5 à $8 \%$ des cas. 50 à $80 \%$ ont des anomalies caryotypiques. Ils ne nécessitent une exérèse qu'en cas de gêne esthétique ou fonctionnelle [1]. La lipomatose peut être rencontrée dans différentes pathologies (lipomatose multiple familiale, lipomatose multiple symétrique, MERRF syndrome ...).

Le syndrome MERRF ou épilepsie myoclonique à fibres rouges déchiquetées a été isolé en 1980 par Fukuhara [2]. La prévalence est de 0,9 cas pour 100000 en Europe. La maladie débute le plus souvent à l'adolescence et son aggravation est progressive [3].

Cette encéphalo-myopathie est caractérisée principalement par une épilepsie myoclonique et peut associer une petite taille, une cardiomyopathie, des signes ORL (surdité neurosensorielle, troubles de la déglutition, dysphonie spasmodique), et des signes ophtalmologiques (atrophie, optique, ophtalmoparésie, rétinopathie pigmentaire) [4]. 3\% des patients présentent une lipomatose [5].

Le syndrome MERRF est lié à une mutation de l'ADN mitochondrial, transmis selon les lois de l'hérédité maternelle, non mendélienne [3]. Le diagnostic prénatal n'est pas conseillé du fait de la possibilité de distribution tissulaire hétérogène de l'hétéroplasmie. Il existe de plus une grande variabilité d'expression intra et interfamiliale qui complique le conseil génétique.

La mutation la plus fréquente est la mutation $8344 \mathrm{~A}>\mathrm{G}$ dans le gène MTTK de transfert de la lysine. Elle concerne plus de $80 \%$ des patients. Une mutation variante $8363 \mathrm{G}>\mathrm{A}$ a également été décrite [3]. Ces mutations altèrent la synthèse des protéines mitochondriales et entraînent une dysfonction de leur chaîne respiratoire. La progression des symptômes peut être expliquée par l'accumulation de cet ADN mitochondrial muté dans les tissus. L'expression clinique de la mutation dépend de plusieurs facteurs: l'hétéroplasmie, la distribution tissulaire et la vulnérabilité tissulaire face au métabolisme oxydatif altéré.

L'association entre syndrome MERRF et lipomatose axiale a été étudiée sur quelques familles [6-7]. Des taux élevés d'ADN mitochondrial muté, une ultrastructure mitochondriale anormale et des anomalies chromosomiques ont été retrouvés dans les lipomes de patients atteints de la mutation $8344 \mathrm{~A}>\mathrm{G}[8,9]$. Dans de rares cas, la seule manifestation clinique de cette mutation était l'apparition d'une lipomatose [8]. La proportion de patients symptomatiques cliniquement augmente avec l'âge [10].

La biopsie musculaire montre la présence de fibres rouges déchiquetées [3].

Il n'y a pas de traitement spécifique. Une prise en charge pluridisciplinaire adaptée aux symptômes est requise [3].

\section{Conclusion}

Devant une lipomatose axiale, des lipomes récidivants ou rapidement extensifs, il est pertinent de rechercher des antécédents personnels ou familiaux d'épilepsie, de myopathie, ou de neuropathie faisant suspecter un syndrome MERRF. Il est également judicieux de réaliser une biopsie musculaire sur la voie d'abord de l'exérèse chirurgicale à la recherche de fibres rouges déchiquetées.

\section{Déclaration de liens d'intérêts}

Les auteurs déclarent ne pas avoir de lien d'intérêt. 


\section{Figure 1}

Imagerie scannographique du lipome géant cervico-dorsal présenté dans l'observation $\mathrm{n}^{\circ} 1$, mesurant $15 \mathrm{~cm}$ de diamètre antéropostérieur et $30 \mathrm{~cm}$ de diamètre transverse.

Coupe axiale (A) et sagittale (B). 


\section{Références}

[1] Delgado L, Ingen-Housz-Oro S. Maladies du tissu adipeux : lipomes, lipomatoses, lipodystrophies. Encyclopédie médico-chirurgicale ;09/09/11. [98-615-A-10]

[2] Fukuhara N, Tokiguchi S, Shirakawa K, Tsubaki, T. Myoclonus epilepsy associated with ragged-red fibres (mitochondrial abnormalities) : disease entity or a syndrome? Light and electron microscopy of two cases and a review of the literature. J Neurol Sci 1980 47:117-33.

[3] DiMauro S, Hirano M. MERRF. SourceGeneReviews® [Internet]. Seattle (WA): University of Washington, Seattle; 1993-2017. 2003 Jun 3 [updated 2015 Jan 29].

[4] Mancuso M, Orsucci D, Angelini C et al. Phenotypic heterogeneity of the 8344A>G mtDNA "MERRF" mutation. Neurology. 2013;80:2049-54.

[5] Hirano M, DiMauro S. Clinical features of mitochondrial myopathies and encephalomyopathies. Lane RJM ed. Handbook of Muscle Disease. New York, NY: Marcel Dekker Inc; 1996. p. 479-504.

[6] Berkovic SF, Shoubridge EA, Andermann F, Andermann E, Carpenter S, Karpati G. Clinical spectrum of mitochondrial DNA mutation at base pair 8344. Lancet. 1991 ; 17;338(8764):457.

[7] Berkovic SF, Carpenter S, Evans A et al. Myoclonus epilepsy and ragged-red fibres (MERRF). A clinical, pathological, biochemical, magnetic resonance spectrographic and positron emission tomographic study. Brain. 1989 Oct;112 ( Pt 5):1231-60.

[8] Holme E, Larsson N G, Oldfors A, Tulinius M, Sahlin P, and Stenman G. Multiple symmetric lipomas with high levels of mtDNA with the tRNA(Lys) A-->G(8344) mutation as the only manifestation of disease in a carrier of myoclonus epilepsy and ragged-red fibers (MERRF) syndrome. Am J Hum Genet. 1993;52(3): 551-6.

[9] Larsson NG, Tulinius MH, Holme E, Oldfors A. Pathogenetic aspects of the A8344G mutation of mitochondrial DNA associated with MERRF syndrome and multiple symmetric lipomas. Muscle Nerve Suppl. 1995;3:S102-6.

[10] Mancuso M, Orsucci D, Angelini C et al. Phenotypic heterogeneity of the 8344A>G mtDNA "MERRF" mutation. Neurology. 2013 ;28;80(22):2049-54 


\section{Figures}

\section{Figure 1}

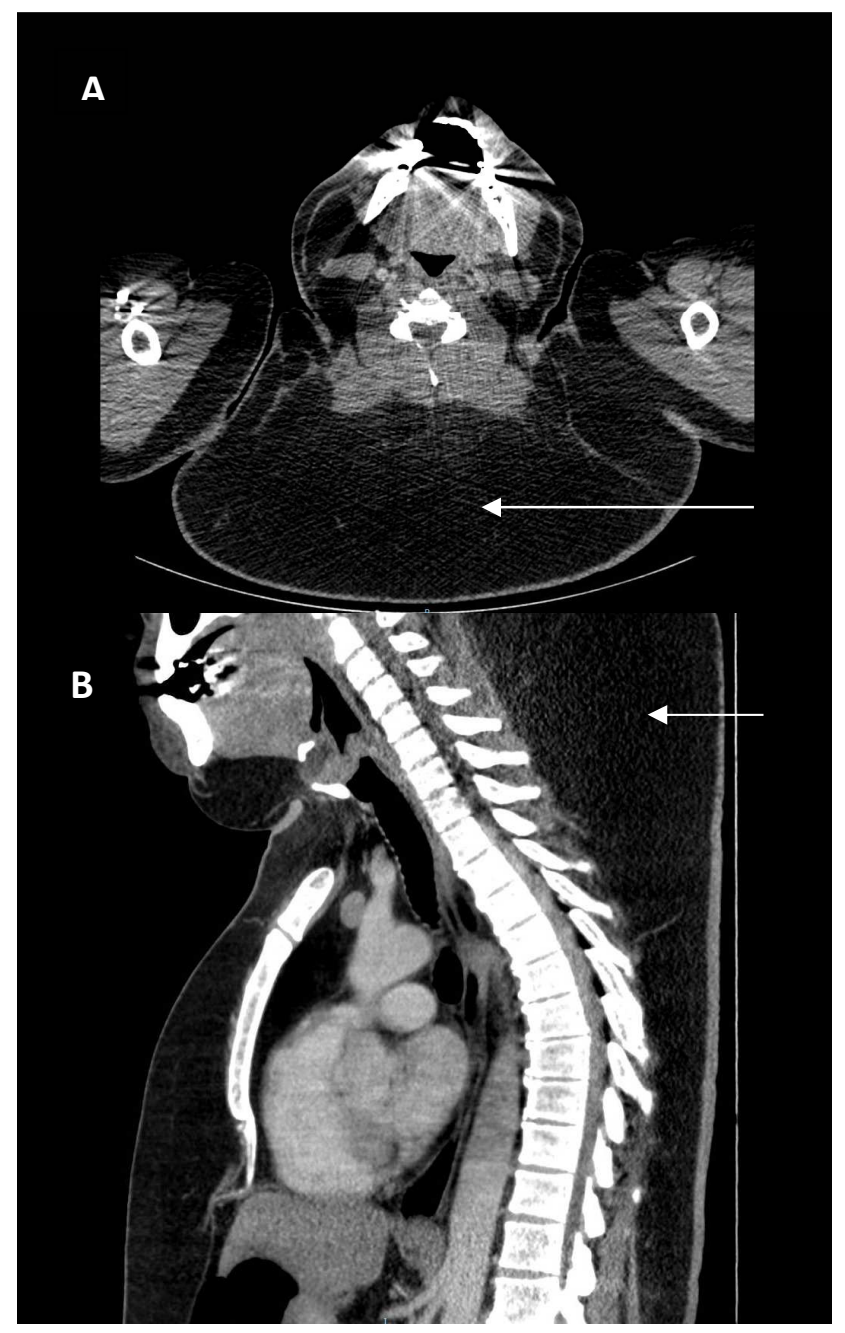

